\title{
Ethical qualms about genetic prognosis
}

\author{
CMAJ Podcasts: author interview at https://soundcloud.com/cmajpodcasts/151320-medsoc
}

$\mathrm{W}$ e believe your genetic information should be controlled by you." Under that banner of individual autonomy, the consumer genetics firm 23 andMe conducted personalized genetic testing until it was stopped in November 2013 by the United States Food and Drug Administration (FDA). The FDA ban occasioned a fierce dispute between proponents of appropriate regulation and defenders of the libertarian view that the only person deciding whether you should have a genetic test should indeed be you. This debate has centred on whether consumers are the best judges of their own clinical care. In this article, I also examine whether the science of personalized medicine is really as advanced as its proponents claim, and how the availability of genetic markers affects decisions on who gets and does not get medical treatment.

In its decision, the FDA cited concerns about inaccuracy arising from the limited sample of markers being tested. Researchers have found that test results from different consumer genetics companies for common diseases, such as diabetes and prostate cancer, can be radically inconsistent because of the low number of markers tested and the small amounts of genetic variance explained by each marker. ${ }^{2}$ In fact, genetic tests offered by direct-toconsumer firms only sample a small proportion of single-nucleotide polymorphisms, (i.e., the points where the genomes of different individuals vary by a single DNA base pair).

In October 2015, 23andMe obtained permission from the FDA to resume a limited version of its genetic testing operations. The company previously covered the risk propensity for 254 inherited diseases; however, it is now only allowed to provide nonmedical ancestry tests and carrier data for 36 conditions, which include sickle cell anemia and cystic fibrosis. It is not permitted to perform pharmacogenetic

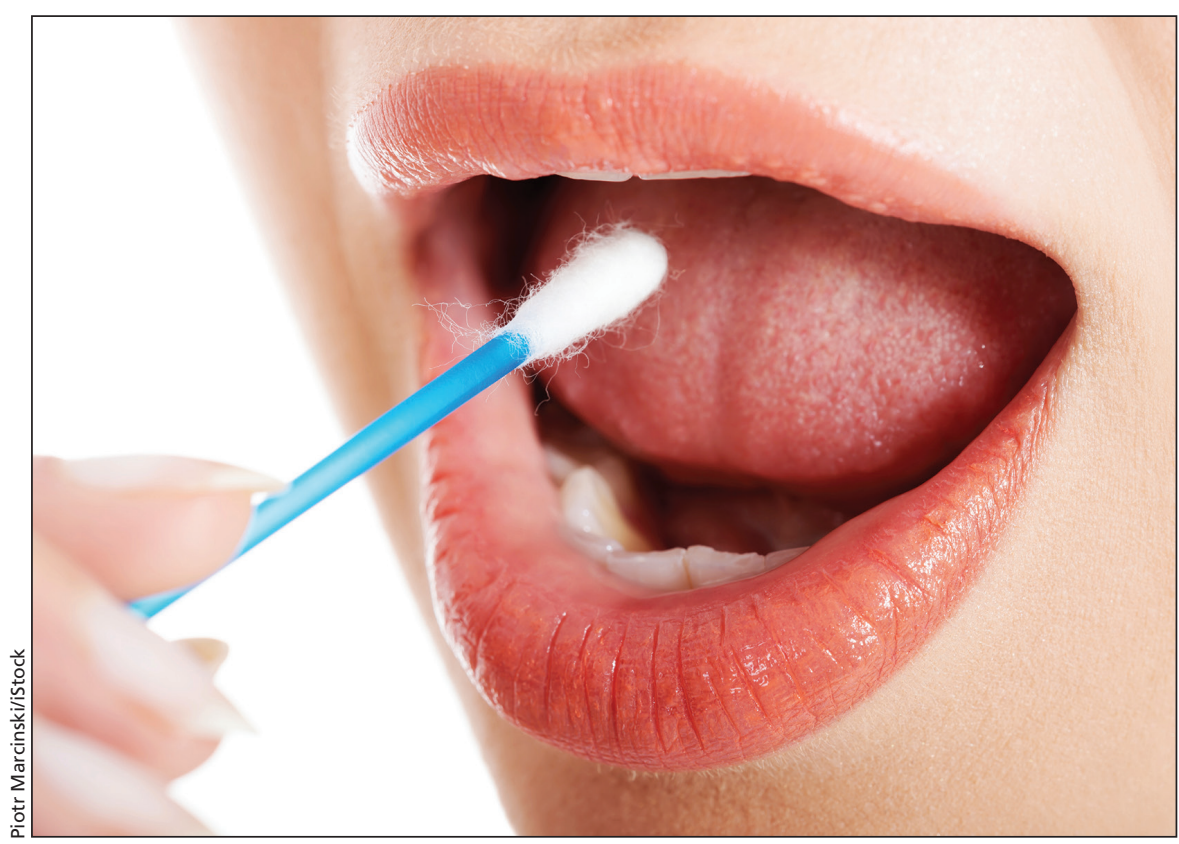

analyses of an individual's tolerance for a particular drug regimen, such as chemotherapy or warfarin.

This final limitation is important, because at the outset, personalized direct-to-consumer genetic testing was widely hailed as the first staging post in the race to develop new forms of medicine that would potentially allow physicians to offer care tailored to the individual's genome rather than the "one-size-fits-all" model. Variously called precision, personalized or stratified medicine, this approach was termed "a true revolution in medicine" by Francis Collins, former co-director of the Human Genome Project, but this assertion is debatable. ${ }^{3}$

Personalized medicine's claim is arguably strongest in the area of pharmacogenetics, the relation of heritable variation to individual differences in drug response. Here, the goal is to minimize adverse drug reactions and increase efficacy by tailoring the drug regimen to the sequenced genomes of both the patient and the cancer. This double approach is crucial because cancer is frequently heterogeneous (e.g., after sequencing the genomes of breast cancers in 50 patients, researchers found that only $10 \%$ of the tumours had more than three mutations in common). ${ }^{4}$

Recent studies have often confirmed or reconfirmed the value of the pharmacogenetic approach. An analysis of 1380 gliomas, involving patients who were classified according to three genetic mutations in the tumours, found that the prevalent histologic approach was less effective than a genomic one for predicting response to treatment and overall prognosis. ${ }^{5}$ A genetic map of prostate cancer in 150 patients, which showed that it comprises several diseases responsive to different treatments, also analyzed the genetic basis for resistance to the standard therapies as a result of specific DNA mutations. ${ }^{6}$

However, other recent studies have revealed serious clinical and ethical limitations of the pharmacogenetic approach. An analysis involving 815 patients with cancer showed that sequencing tumour genomes for clues to genetic changes can actually misdirect individualized treatment in nearly half of patients, unless this sequencing 
is first compared with a genetic readout of the patient's noncancerous tissue. Two-thirds of genetic changes identified with tumour-only sequencing were false-positive results, which means patients might be given the wrong targeted treatments. ${ }^{\text {? }}$

This study undermines the standard argument about resource allocation and personalized medicine: even though pharmacogenetic drugs are frequently more expensive, this form of personalized medicine is more cost-effective because only those patients who would benefit from treatment receive it. The ethos of pharmacogenetics is "the right treatment for the right patient at the right time." However, such a high rate of false-positive results could mean the wrong treatment for the "right" patient.

In other cases, genetic testing might result in some patients being denied treatment, which raises ethical issues about resource allocation. For example, genetic markers can predict the probability and severity of immune reaction in kidney transplant patients. ${ }^{8}$ Would it be ethical to select potential kidney recipients primarily or solely on a genetic basis? Although individualized genetic testing does not create the ethical dilemma arising from the need to make choices - because of the shortage of donor kidneys - it does not dispel it either.

Arguably, it is more efficient and equitable to use advances in genetic sequencing to maximize the chances that the scarce resource of a donated kidney will be put to successful use. But we must also confront the problem of false-negative results, the mirror image of the false-positive problem raised by the study on prostate cancer. Suppose a potential recipient is wrongly identified as genetically more likely to reject the kidney and is disqualified for the transplant on that basis. This problem is exacerbated by the probabilistic nature of genetic analysis, which rarely yields black-and-white answers. Should a patient get a kidney, or a drug, if there is only a $20 \%$ chance that he or she will respond favourably based on genetic profile? Ten per cent? One per cent? Where shall we draw the line?

The ethics of resource allocation, that area of "tragic choices" in medicine, offers two main alternatives for answering such questions. We can select who shall receive a scarce resource on the basis of prognosis: giving the kidney or drug to the patient with the best chance of recovery. Such systems of triage have long been used by medicine, although they are sometimes controversial. Choosing on the basis of the genetic likelihood of immune system rejection is a variant of this prognosis-based approach. Of course, in a clinical context, many other factors (e.g., patient age and family history) are also likely to be considered and will probably provide more accurate information than personalized genetic screening. ${ }^{10}$

The alternative model of resource allocation is by diagnosis. Here, the argument is that justice demands we should give the resource to those with the most pressing medical need, not the best predicted outcome. We often favour this second criterion. For example, in palliative care we think that dying patients should receive more medical resources than their terminal prognosis would dictate. Similarly, we might want to give the patient with no other alternative an expensive pharmacogenetic drug, even if his or her genetically ascertained prognosis for the drug's likely success is less favourable than in other patients.

As a medical ethicist, I would have qualms about dividing patients into treatment and nontreatment camps purely on the basis of a genetic prognosis, particularly when genetic prognosis is still such an inexact science. A patient who has no other alternative may want to take his or her chances with a pharmacogenetic drug. Although personalized medicine is framed in the language of individual rights and autonomy, resource allocation decisions are ultimately made by others. Particularly in an era of austerity and cost-cutting, the possibility that decision-makers will use genetic testing to limit patients' "right to choose" is far from remote.

\section{Donna Dickenson MSc PhD}

Emeritus Professor of Medical Ethics and Humanities, University of London, London, UK

Author of a critical study of personalized medicine, Me Medicine vs. We Medicine (Columbia University Press, 2016)

\section{References}

1. McGowan ML, Fishman JR, Lambrix MA. Personal genetics and individual identities: motivations and imperatives of early users. New Genet Soc 2010;29:261-90.

2. Kalf RRJ, Mihaescu R, de Knijff P, et al. Risk predictions from direct-to-consumer personal genetic testing: What do consumers really learn about common diseases? Paper presented at the European Society for Human Research and Enbryology (ESHRE) Conference; 2009 May 29-30.

3. Collins FS. The language of life: DNA and the revolution in personalized medicine. New York: Harper Collins, 2010:29.

4. Wadman M. Fifty genome sequences reveal breast cancer's complexity [online]. Nature 2011 Apr. 2. doi:10.1038/news.2011.203.

5. Eckel-Passow JE, Lachance DH, Molinaro AM, et al. Glioma groups based on $1 \mathrm{p} / 19 \mathrm{q}$, IDH and TERT promoter mutations in tumours. $N$ Engl $J$ Med 2015;372:2499-508.

6. Robinson D, Van Allen EM, Wu Y-M, et al. Integrative clinical genomics of advanced prostate cancer. Cell 2015;161:1215-28.

7. Jones S, Anagnostou V, Lytle K, et al. Personalized genomic analysis for cancer mutation discovery and interpretation. Sci Transl Med 2015;7:283ra53.

8. Dion-Labrie M, Fortin MC, Hebert MJ. The use of personalized medicine for patient selection for renal transplantation: physicians' views on the clinical and ethical implications. BMC Med Ethics 2010;11:5.

9. Calabresi G, Bobbitt P. Tragic choices. New York: WW Norton; 1978.

10. Heald B, Edelman E, Eng C. Prospective comparison of family medical history with personal genome screening for risk assessment of common cancers. Eur J Hum Genet 2012;20:547-51.

CMAJ 2016. DOI:10.1503/cmaj.151320 